\title{
Authentication, Micromorphology and Ultrastructure of Pollen Grains and Seeds of Endemic Taxa in Saint Katherine Protectorate, South Sinai, Egypt
}

\author{
Mohamed M. El-Khalafy ${ }^{1 *}$, Yassin M. Al-Sodany ${ }^{1}$, Kamal H. Shaltout ${ }^{2}$, Soliman A. Haroun ${ }^{1}$, Dalia A. Ahmed ${ }^{2}$ and $^{2}$ \\ Mohamed A. Salim ${ }^{3}$ \\ ${ }^{1}$ Botany and Microbiology Department, Faculty of Science, Kafrelsheikh University, Kafr Elsheikh, Egypt \\ ${ }^{2}$ Botany Department, Faculty of Science, Tanta University, Tanta, Egypt \\ ${ }^{3}$ Botany Department, Faculty of Science, Ain Shams University, Cairo, Egypt \\ *For correspondence: mohamed_elkhalfy91@sci.kfs.edu.eg \\ Received 05 April 2021; Accepted 11 June 2021; Published 10 July 2021
}

\begin{abstract}
The endemic taxa were restricted to a specific geographic region and they are essential for setting conservation priorities. This study aimed to update the endemic taxa list in Saint Katherine Protectorate (SKP) depending on literature reviews, field trips and herbaria consultation. Other characters also recorded viz, sex forms, dispersal types and flowering time. Also, the morphological characters of the pollen grains and seeds were examined and photographed using light microscope (LM) and scanning electron microscope (SEM). In addition, the mineral composition of pollens and seeds was detected using energy dispersive X-ray (EDX). The updated list included 13 taxa belonging to 11 genera and 8 families. All the recorded taxa were bisexual; ballochores were the most represented dispersal type. There was a gradual increase in the endemic taxa from March to August while decreasing from October to February. Pollens were isopolar and medium in size. They possessed colpate, colporate, or porate aperatus, as well as reticulate exine sculpture. Furthermore, operculum and margo were absent in most of the pollens. The seed colour ranged from light brown to black; elliptic; basal hilum; polygonal and irregular-shaped seeds were the most represented. All previous characters were diagnosed at generic and specific levels, which helped in the construction of artificial keys to facilitate the differentiation between the studied taxa. The present study has the priority in describing pollens and seeds of Astragalus fresenii and Micromeria serbaliana, in addition to the description of the seeds of Ballota kaiseri. The presence and percentage of twelve elements detected by EDX differed significantly within the investigated pollen grains and seeds. The present data indicated that pollen grains and seeds of studied taxa had high percentages of carbon, oxygen, phosphorous, magnesium, nitrogen and calcium. This study is the first attempt using EDX technique with these taxa. (C) 2021 Friends Science Publishers
\end{abstract}

Keywords: Authentication; EDX; Endemic; Pollen morphology; Saint Katherine; Seed morphology

\section{Introduction}

The endemic taxa inhabit particular habitats restricted to a specific area due to factors such as isolation, climatic changes or urban development. Such species were often endangered, so it is essential to conserve them (Brooks et al. 2006; Lima et al. 2020). Egypt is divided into four geographic regions; Nile, Western Desert, Eastern Desert and Sinai Peninsula. The latter possesses a unique triangular shape and all the geologic formations, structures, and landforms of Egypt are nearly represented in it. Moreover, Saint Katherine Protectorate (SKP) encloses most of the mountainous area of South Sinai and occupies about 4350 $\mathrm{km}^{2}$ (Embabi 2018).

The SKP is extremely arid with long, hot, rainless summers and cold, rainy winters and lies in the low rain belt of Egypt with an annual rainfall of $57 \mathrm{~mm} /$ year. However, its high mountains receive higher amounts of precipitation (100 mm/year) as rain and sometimes snow. Nonetheless, rainfall is not an annual characteristic rather two to three consecutive years without rainfall is common. Rain takes the form of sporadic flash floods or limited local showers; thus, high spatial heterogeneity in receiving moisture is also common (Ibraheem 2012).

Danin (1986), reported 28 endemic taxa in Sinai, 25 of them occur in the mountainous district. Täckholm (1974) reported 34 taxa restricted to Sinai out of 69 endemic taxa in Egypt. According to El-Hadidi and Fayed (1994/1995), 27 taxa endemic to South Sinai were reported, while El-Hadidi and Hosni (2000) recorded 30

To cite this paper: El-Khalafy MM, YM Al-Sodany, KH Shaltout, SA Haroun, DA Ahmed and MA Salim (2021). Authentication, micromorphology and ultrastructure of pollen grains and seeds of endemic taxa in saint katherine protectorate, South Sinai, Egypt. Intl J Agric Biol 26:359-370 
endemic taxa to Sinai as a whole. Boulos (2009) reported 20 endemic taxa in SKP out of 31 taxa in Sinai. Hosni et al. (2013) reported 17 taxa in SKP out of 27 taxa in Sinai. Zahran et al. (2015) reported 17 endemic taxa in SKP out of 28 taxa in Sinai. At the same time, Abdelaal et al. (2018) reported 14 taxa in SKP out of 26 taxa in Sinai. Generally, the endemic taxa in SKP are growing in four habitats; Wadi bed, gorge, slope and basin. The gorge habitat is the most suitable for the growth of endemic taxa, while the basin is the lowest (Zahran et al. 2015).

Palynology is a fascinating science with a multidimensional approach covering almost botanical science branches and is considered a helpful tool in taxonomic and evolutionary studies. Many successful trials taken pollen characters as a basis for classification (Erdtman 1952; Takhtajan 1980). Pollen morphology helped botanists and ecologists to reconstruct the past assemblages of plants and identify periods of environmental change (Krzywinski et al. 1989; Moore et al. 1991; Yao et al. 2017).

Seed morphological characters are considered a powerful tool for the classification of various plant groups. These characters, especially the seed shape and details of the outer seed coat's sculpturing can be quite variable and important compared to other organs, to cite but a few, we can refer to (Barthlott 1981, 1984; Svetlova 2008; Lomonosova 2009; Kaya et al. 2016). This is because the seed and fruit characteristics are less affected by changing the environmental conditions (Zoric et al. 2010).

The research studies on the morphology of pollen grains and seeds of endemic taxa in Saint Katherine are few. There is no previous complete data available, despite that we can refer to some studies dealt with these taxa either in the context of the endemic flora of Egypt or as separate taxa within special groups (Shehata and Kamel 2007; Rabei et al. 2016; El-Ghamery et al. 2018; Shiha 2020).

Energy descriptive X-ray spectroscopy (EDX) provides a quantitative and qualitative element analysis of the samples. One advantage of this technique is that tiny specimens (such as pollen grains or seeds) can be analyzed under electron beam control. EDX depends on the type of $\mathrm{X}$-ray called the characteristic X-ray; produced from samples due to the electron beam interaction. The released $\mathrm{X}$-rays exhibit a pattern of peaks at energy corresponding to the element. An EDX analysis system combined with a SEM allows investigating of the elemental composition of biological samples. The elemental composition of pollen can be measured without destroying the organic matrix (Lott et al. 1982; Lott 1984; Ockenden and Lott 1988). Pocock and Vasanthy (1986) think that elements detected in the pollen grains with EDX depend on the percentage of the component in the soil where the plants were grown. Wolter and Nilsson (1990) used the EDX investigations on the pollen of some taxa belonging to Apocynaceae family for systematic purposes. Rehman et al. (2008) used EDX to detect the potassium accumulation in the sulcus area in pollen of Pinus densiflora. The present study is the first attempt to use this technique with these taxa.

Most of the endemic taxa have become closer to extinction within the last years due to environmental conditions and human activities. Hence, the goal of the present study was to share the knowledge of endemic taxa in SKP including investigation of pollen grains and seeds morphological characters (macro- and micro-characters), and the element detection by using EDX, constructing artificial keys based on the most prominent characters for identifying and discriminating between the studied taxa.

\section{Materials and Methods}

\section{Field trips}

Twenty field trips were conducted from summer 2017 to spring 2020 to SKP for collecting the studied taxa as fresh materials from their natural habitats (Fig. 1). Regarding Astragalus fresenii, we depended on a herbarium specimen from Cairo University Herbarium (CAI). Samples of flowers and seeds were collected for laboratory investigations. Identification and synonyms followed Täckholm (1974), Boulos (1999-2005, 2009) and International Plant Names Index (http://www.ipni.org). The collected taxa were compared with holotypes preserved at different herbaria in Egypt. The affiliation of taxa to their families followed APG (2016). Voucher specimens were preserved in the herbarium of Botany and Microbiology Department, Faculty of Science, Kafrelsheikh University and the herbarium of Botany Department, Faculty of Science, Tanta University (TANE), Egypt.

Authentification and verification of the endemic taxa in SKP were carried out according to: Täckholm and Täckholm (1941); Täckholm and Drar (1950-1969); Täckholm (1974); Boulos (1999-2005, 2009); El-Hadidi and Hosni (2000); Taifour and El-Oqlah (2014); Ibrahim et al. (2016); El-Khalafy (2018); Shaltout et al. (2018) and Abdelaal et al. (2018). Some websites were consulted to collect more information about the recorded taxa (Table 1). Gaps of information collected from the herbaria of Cairo University (CAI), Ain Shams University (CAIA), Agriculture Museum (CAIM), Tanta University (TANE), Assiut University (ASTU), Aswan University (ASW) and Alexandria University (ALEX).

\section{Sex form, flowering time and dispersal types}

Sex forms were determined directly from the flowers in the field. Flowering time was determined according to field observations and data from preserved herbarium sheets. Dispersal types were recorded according to description of dispersal units depending on the available diaspores and the studies of Täckholm and Täckholm (1941); Täckholm and Drar (1950-1969) and Boulos (1999-2005). 
Table 1: Global online databases used for authentication of the endemic taxa in the SKP

\begin{tabular}{lll}
\hline No. & Database & Source \\
\hline 1 & International Plant Names Index (IPNI) & http://www.ipni.org \\
2 & The Plant List (TPL) & http://www.theplantlist.org \\
3 & Plants of the World Online (POWO) & http://www.plantsoftheworldonline.org \\
4 & Catalogue of Life & http://www.catalogueoflife.org/annual-checklist/2010 \\
5 & Tropicos & http://www.tropicos.org \\
6 & Global Biodiversity Information Facility (GBIF) & http://www.gbif.org/occurence \\
7 & JSTOR Global Plants & http://plants.jstor.org \\
8 & Euro+Med PlantBase & http://ww2.bgbm.org/EuroPlusMed/query.asp \\
9 & Kew World Checklist of Selected Plant Families (WCSP) & http://wcsp.science.kew.org \\
10 & King Saud University & http://faculty.ksu.edu.sa \\
11 & Asian Flora Database & http://www.asianflora.com \\
12 & Flora of Israel Online & http://flora.org.il \\
13 & India Biodiversity Portal & https://indiabiodiversity.org/species/show/245963
\end{tabular}

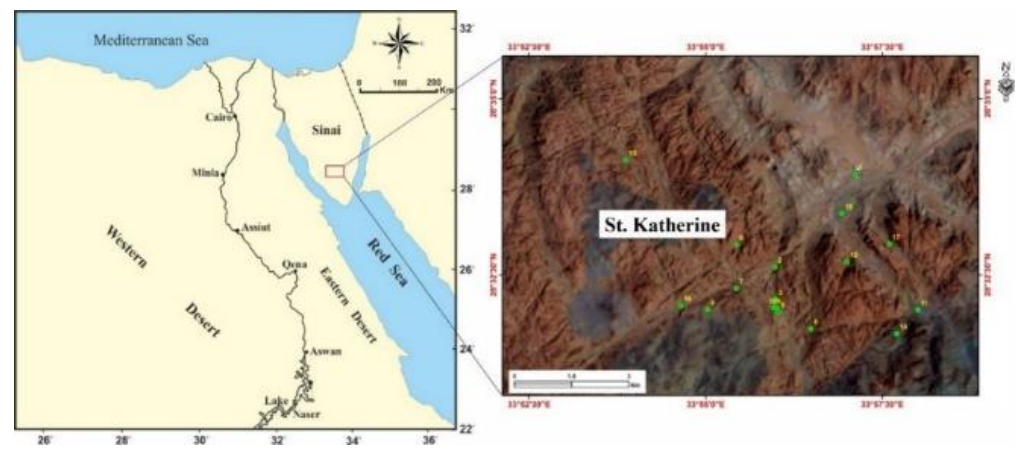

Fig. 1: Map of Saint Katharine Protectorate showing the visiting sites

\section{Pollen grain morphology}

Flowers of the studied taxa were fixed in $70 \%$ ethanol. For LM investigation, mature anthers from the collected flowers were left to dry, carefully opened using sharp needles and sputtered onto glass slides. The pollen grains (five to ten pollen grains per taxa) were examined, measured and photographed using Canon power-shot A470, 7.1 megapixels. The polar axis $(\mathrm{P})$ and equatorial diameter $(\mathrm{E})$ were measured, pollen size, shape and aperture type were also assessed. For SEM investigation, non-acetolyzed pollen grains were transferred onto a metallic stub using a doublesided cellotape and coated with a thin layer of gold in a sputtering chamber, then scanned and photographed using JEOL JSM-IT100 SEM for exine and aperture ornamentations. The terminology used for describing pollen grains morphology was followed as given by Erdtman (1952); Punt et al. (2007) and Hesse et al. (2009).

\section{Seed morphology}

Mature seeds (five to ten seeds from each taxon) were examined using stereo-microscope and photographed using Canon power-shot A470, 7.1 mega pixels digital camera. Stage micrometer in addition to ImageJ software was used for seed measurements and calibration. Seed color, length and width, as well as hilum position were studied by stereomicroscope. For SEM investigation, the mature seeds were mounted onto SM stubs, coated with gold and examined and photographed using JEOL JSM-IT100 SEM. In Rosa arabica the achene must be opened and its wall shed off to allow the seed's appearance. Terminology was followed as given by Barthlott (1981) and Stearn (1992). The SEM photographs were carried out in the Institute of Nanoscience and Nanotechnology, Kafrelsheikh University.

\section{Energy descriptive X- ray technology (EDX)}

The non-acetolyzed pollen grains and mature seeds were placed in a JEOL JSM-IT100 scanning electron microscope equipped with an EDX detector at the Institute of Nanoscience and Nanotechnology, Kafrelsheikh University and coated with a very thin film of gold. The beam was focused on pollen and seed at $20 \mathrm{KV}$ and analyzed for approximately 20 seconds and the machine dead time was not included. The count rate per second (CPS) reached 1845.

\section{Results}

\section{Recorded taxa}

Thirteen endemic taxa (11 species, one subspecies and one variety) belonging to 11 genera and eight dicotyledonous families were recorded. Caryophyllaceae was the most represented family (four taxa), followed by Lamiaceae (three taxa). Silene was the most represented genus (three taxa), while the remaining genera were represented by only 
Table 2: Collection data, dispersal type and flowering time of the studied taxa in SKP

\begin{tabular}{|c|c|c|c|c|}
\hline No. & Taxa & Family & Dispersal Type & Flowering time \\
\hline 1 & $\begin{array}{l}\text { Anarrhinum pubescens Fresen } \\
=\text { Anarrhinum duriminium } \text { (Brot.) Pers } \\
=\text { Anarrhinum forsskaohlii (J. F. Gmel.) Cufod. subsp. pubescens (Fresen.) D. A. Sutton } \\
=\text { Anarrhinum orientale Benth. var. pubescens (Fresen.) Rouy } \\
=\text { Cardiotheca pubescens } \text { (Fresen.) Ehrenb. ex Steud. } \\
=\text { Simbuleta pubescens } \text { Kuntze }\end{array}$ & Scrophulariaceae & Ballochore & March - May \\
\hline 2 & $\begin{array}{l}\text { Astragalus fresenii Decne } \\
=\text { Tragacantha fresenii } \text { (Decne.) Kuntze }\end{array}$ & Fabaceae & Pogonochore & March- May \\
\hline 3 & Ballota kaiseri Täckh & Lamiaceae & Microsclerochore & March-May \\
\hline 4 & Buffonia multiceps Decne & Caryophyllaceae & Ballochore & March - June \\
\hline 5 & $\begin{array}{l}\text { Hyoscyamus boveanus (Dunal) Asch. \& Schweinf } \\
=\text { Scopolia boveana } \text { Dunal }\end{array}$ & Solanaceae & Ballochore & March - June \\
\hline 6 & $\begin{array}{l}\text { Micromeria serbaliana } \text { Danin \& Hedge } \\
=\text { Satureja serbaliana } \text { (Danin \& Hedge) Greuter \& Burdet }\end{array}$ & Lamiaceae & Microsclerochore & April- August \\
\hline 7 & $\begin{array}{l}\text { Origanum syriacum } \text { L. subsp. sinaicum } \text { (Boiss.) Greuter \& Burdet } \\
=\text { Origanum maru } \text { L. var. sinaicum Boiss. } \\
=\text { Majorana nervosa } \text { Benth. } \\
=\text { Origanum syriacum } \text { var. sinaicum } \text { (Boiss.) Ietsw. } \\
=\text { Origanum nervosum } \text { (Benth.) Vogel } \\
=\text { Majorana syriaca } \text { (L.) Raf., nom. illegit. }\end{array}$ & Lamiaceae & Microsclerochore & April- October \\
\hline 8 & $\begin{array}{l}\text { Polygala sinaica } \text { Botsch. var. sinaica } \\
=\text { Polygala spinescens Decne }\end{array}$ & Polygalaceae & Pogonochore & April- August \\
\hline 9 & $\begin{array}{l}\text { Primula boveana Decne. ex Duby } \\
=\text { Primula verticillata } \text { subsp. boveana (Decne.) W.W. Sm. \& Forrest } \\
=\text { Primula verticillata } \text { Forssk. }\end{array}$ & Primulaceae & Ballochore & March - June \\
\hline 10 & $\begin{array}{l}\text { Rosa arabica } \text { Crép. } \\
=\text { Rosa rubiginosa } \text { var. arabica } \text { (Crepin) Boiss } \\
=\text { Rosa rubiginos } \mathrm{L} . \\
=\text { Rosa agrestis } \text { Savi }\end{array}$ & Rosaceae & Ballochore & June- August \\
\hline 11 & Silene leucophylla Boiss & Caryophyllaceae & Ballochore & May- June \\
\hline 12 & $\begin{array}{l}\text { Silene oreosinaica } \text { Chowdhuri } \\
=\text { Silene sinaica } \text { Boiss }\end{array}$ & Caryophyllaceae & Ballochore & March- April \\
\hline 13 & Silene schimperiana Boiss & Caryophyllaceae & Ballochore & March- August \\
\hline
\end{tabular}

one taxon (Table 2). All the studied taxa were bisexual (hermaphrodites). Ballochore (seven taxa $=53.8 \%$ of the total taxa) was the most represented dispersal type, followed by microsclerochore (three taxa $=23.1 \%$ ) and pogonochore (two taxa $=15.4 \%$ ). There was a gradual increase in the frequency of flowered taxa from March till reaching a maximum in April and May (12 taxa $=92.3 \%)$. In general, the period from March to May was characterized by the highest, while the period from September to February was characterized by the lowest flowering activity (Table 2).

\section{Pollen morphology}

The examined pollen grains showed considerable variations in their characteristics as they represented different genera and families. The polar length ranged from 14.72 to $44.51 \mu \mathrm{m}$, while the equatorial length ranged from 9.31 to $45.04 \mu \mathrm{m}$. In general, medium size was the most represented. Most of the pollen grains were isopolar. The apertures were colpate, colporate or porate. Operculum and margo were absent in most of the studied taxa. Eight types of exine sculpture were described; reticulate type was the most represented (four taxa). The annulus was absent in most of the studied taxa. The pollen morphological characters (LM and SEM) were summarized in Table 3, and some of the specific structures (micro-photographs) were arranged and illustrated in Fig. 2. So far as the data of the present work are concerned, the subsequent artificial key based on the pollen morphological characters is provided to enable the different endemic taxa to SKP to be distinguished.

\begin{tabular}{ll}
\hline Distinguishing features & Character/taxa \\
\hline 1a. Apolar pantoporate pollen & 2 \\
1b. Isopolar colpate or colporate & 5 \\
2a. Operculum present & 3 \\
2b. Operculum absent & 4 \\
3a. aculeate exine sculpture & Buffonia multiceps \\
3b. Foveate- micropapilate exine sculpture & Silene oreosinaica \\
4a. 25- 30 pore/ grain, tectate & Silene leucophylla \\
4b. 15- 20 pore/ grain, tectum indistinct & Silene schimperiana \\
5a. Colpate & 6 \\
5b. Colporate & 9 \\
6a. Stephanocoplate(22-27) pore/pollen, psilate exine sculpture & Polygala sinaica \\
6b. Tri- or hexacolpate & 7 \\
7a. Tricolpate, reticulate perforate pollen & Ballota kaiseri \\
7b. Hexacolpate & 8 \\
8a. Medium- sized, prolate grain & Micromeria serbaliana \\
8b. Small- sized, subprolate grain & Origanum \\
& sybsiacum \\
9a. Subprolate, reticulate exine sculpture & Astragalus fresenii \\
9b. Prolate or prolate- spheroidal pollen & 10 \\
10a. Prolate, reticulate- foveolate exine sculpture & Anarrhinum pubescens \\
10b. Prolate- spheroidal pollen & 11 \\
11a. Large- sized pollen, striate- perforate exine sculpture & Hyoscyamus boveanus \\
11b. Medium- sized pollen & 12 \\
12a. Reticulate exine sculpture & Primula boveana \\
12b. Striate exine sculpture & Rosa arabica \\
\hline
\end{tabular}

\section{Seed morphology}

The examined seeds showed considerable variations in their characteristics as they represented different genera and families. The color of the seeds ranged from light brown to black. Seven shapes of seeds were described; elliptic type was the most represented (four taxa). The hilum position for most taxa was basal, while its level may be raised, flat, 


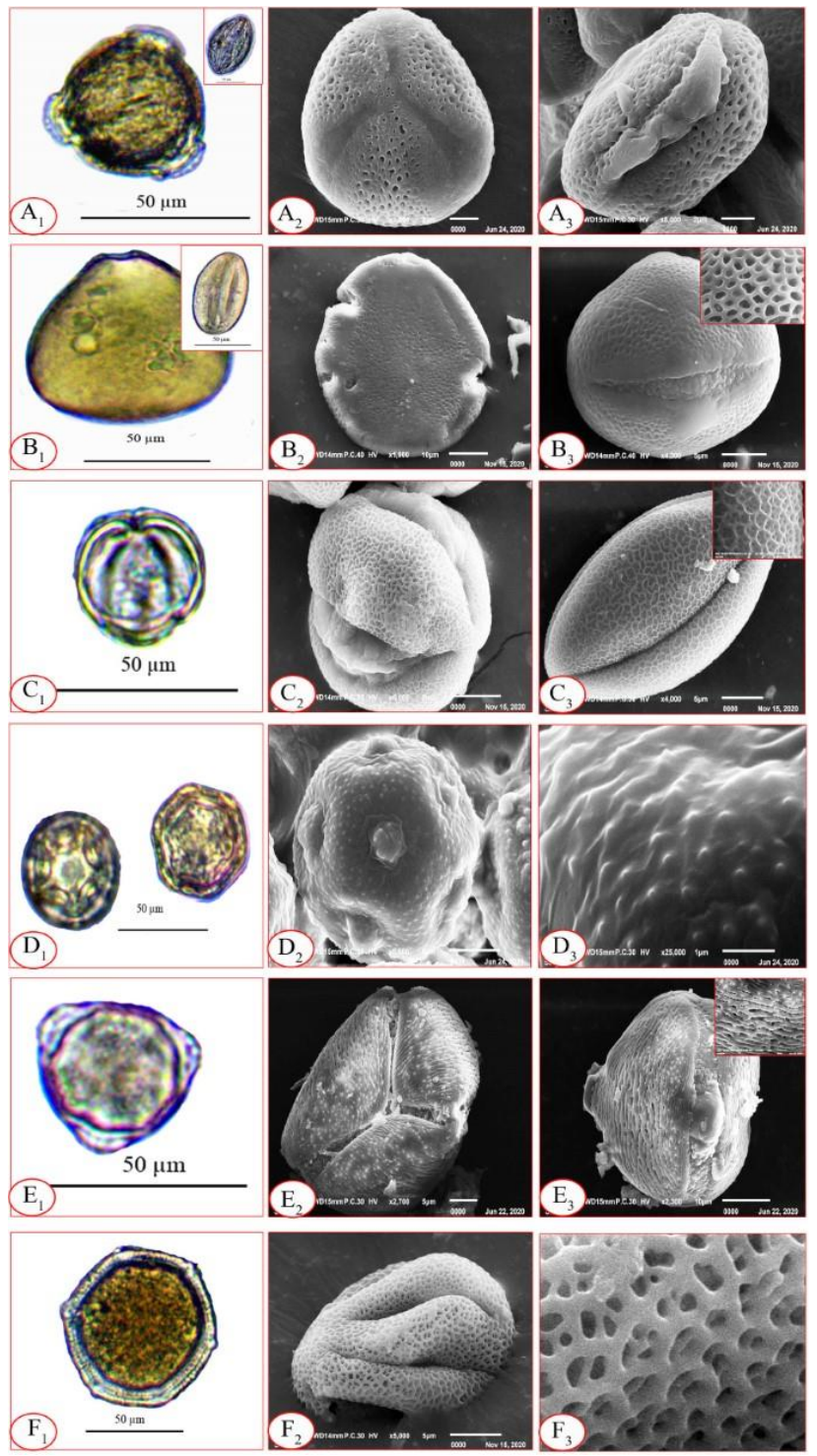

Fig. 2: A-F. Micro-photographs of pollen grains of the studies taxa (LM \& SEM). (A) Anarrhinum pubescens; prolate, trizonocolporate, semi-tectate, reticulate- foveolate sculpture, (B) Astragalus fresenii; subprolate, trizonocolporate, tectate, reticulate-sculpture, (C) Ballota kaiseri; prolate, trizonocolpate, tectate, reticulate- perforate sculpture, (D) Buffonia multiceps; prolate-spheroidal, pantoporate, semi-tectate, distinct operculum, aculeate sculpture, (E) Hyoscyamus boveanus; prolate-spheroidal, trizonocolporate, striate- perforate sculpture, (F) Micromeria serbaliana; prolate, hexazonocolpate, tectate, reticulate sculpture

depressed or semi-depressed. Eleven different types of surface sculpture patterns were described. Four types of epidermal cell shapes were described; polygonal and irregular shapes were the most represented. The anticlinal wall may be raised or depressed; its shape may be striate, sinuate or undulate. The seed morphological characters (LM and SEM) were summarized in Table 4 and some of the specific structures (micro-photographs) were arranged and

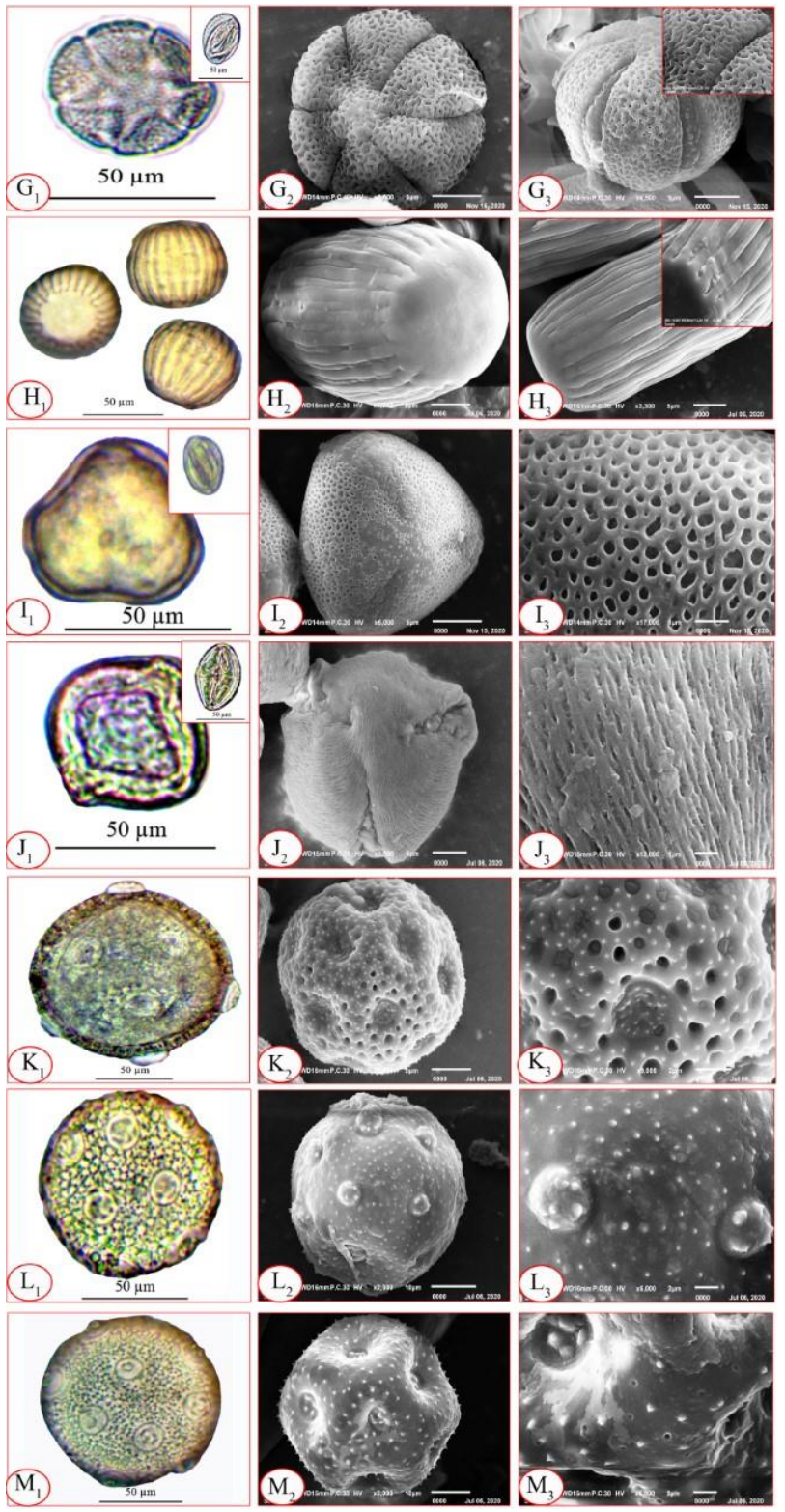

Fig. 2: Cont. G-M. Micro-photographs of pollen grains of the studies taxa (LM \& SEM). (G) Origanum syriacum subsp. sinaicum; subprolate, hexazonocolpate, tectate, reticulate sculpture, (H) Polygala sinaica; prolate, stephanozonocolpate, psilate sculpture, (I) Primula boveana; prolate-spheroidal, trizonocolporate, tectate, reticulate sculpture, (J) Rosa arabica; prolate-spheroidal, trizonocolporate, semi-tectate, striate sculpture, (K) Silene leucophylla; prolate-spheroidal, pantoporate, tectate, foveate- micropapilate sculpture, (L) S. oreosinaica; prolate-spheroidal, pantoporate, semi-tectate, distinct operculum, foveate- micropapilate sculpture, (M) S. schimperiana; prolatespheroidal, pantoporate, foveate- micropapilate sculpture

illustrated in Fig. 3. The seed morphology (macro- or microcharacters) is an additional tool to taxa delimitation as they have a diagnostic value and sometimes the seed characters alone are satisfactory, so an artificial key based on the most 
El-Khalafy et al. / Intl J Agric Biol, Vol 26, No 2, 2021

Table 3: Quantitative and qualitative pollen grains micro-morphological characteristics of the studied taxa (LM \& SEM)

\begin{tabular}{|c|c|c|c|c|c|c|c|c|c|c|c|c|c|c|}
\hline \multirow{2}{*}{$\begin{array}{l}\text { Attributes } \\
\text { Taxa }\end{array}$} & \multicolumn{2}{|c|}{ Dimension $(\mu \mathrm{m})$} & \multirow{2}{*}{$\begin{array}{l}\text { Pollen } \\
\text { size } \\
(\mathrm{P}+\mathrm{E} / 2) \\
\end{array}$} & \multicolumn{2}{|c|}{ Pollen shape } & \multirow[t]{2}{*}{ Polarity } & \multicolumn{4}{|c|}{ Aperture } & \multirow{2}{*}{ Exine sculpture } & \multirow[t]{2}{*}{ Tectum } & \multirow{2}{*}{ Annulus } & \multirow{2}{*}{$\begin{array}{l}\text { Collumella } \\
\text { visibility }\end{array}$} \\
\hline & $\begin{array}{l}\text { Polar = length } \\
\text { (P) }\end{array}$ & $\begin{array}{l}\text { Epuatorial = } \\
\text { width }(\mathrm{E})\end{array}$ & & $\begin{array}{l}\text { Amb } \\
\text { (LM) }\end{array}$ & $\begin{array}{l}\text { Shape class } \\
\text { (P/E X 100) }\end{array}$ & & Shape & Number & Operculum & Margo & & & & \\
\hline 1 & $\begin{array}{l}14.173-16.262 \\
(14.717)\end{array}$ & $\begin{array}{l}8.876-9.756 \\
(9.3115)\end{array}$ & Small & Triangular & Prolate & Isopolar & $\begin{array}{l}\text { Zonocolporate } \\
\text { (elliptic) }\end{array}$ & 3 & Absent & Present & $\begin{array}{l}\text { Reticulate- } \\
\text { foveolate }\end{array}$ & $\begin{array}{l}\text { Semi- } \\
\text { tectate }\end{array}$ & Absent & Indistinct \\
\hline 2 & $\begin{array}{l}22.508-23.409 \\
(22.958)\end{array}$ & $\begin{array}{l}17.26-18.181 \\
(17.720)\end{array}$ & Medium & /l & Subprolate & /I & // & /I & // & /I & Reticulate & Tectate & $/ /$ & Distinct \\
\hline 3 & $\begin{array}{l}30.854-32.747 \\
(31.8)\end{array}$ & $\begin{array}{l}17.113-19.576 \\
(18.344)\end{array}$ & /I & /I & Prolate & /I & $\begin{array}{l}\text { Zonocolpate } \\
\text { (slit- like) }\end{array}$ & /I & /I & Absent & $\begin{array}{l}\text { Reticulate- } \\
\text { perforate }\end{array}$ & /I & // & /I \\
\hline 4 & $\begin{array}{l}18.523-19.436 \\
(18.979)\end{array}$ & $\begin{array}{l}17.642-18.612 \\
(18.127)\end{array}$ & /I & Circular & $\begin{array}{l}\text { Prolate- } \\
\text { spheroidal }\end{array}$ & Apolar & $\begin{array}{l}\text { Pantoporate } \\
\text { (circular) }\end{array}$ & $10-15$ & Present & /I & Aculeate & $\begin{array}{l}\text { Semi- } \\
\text { tectate }\end{array}$ & Present & Indistinct \\
\hline 5 & $\begin{array}{l}37.141-39.26 \\
(38.2)\end{array}$ & $\begin{array}{l}33.02-35.056 \\
(34.038)\end{array}$ & Large & Triangular & // & Isopolar & $\begin{array}{l}\text { Zonocolporate } \\
\text { (elliptic) }\end{array}$ & 3 & Absent & /I & Striate- perforate & Indistinct & Absent & /I \\
\hline 6 & $\begin{array}{l}24.472-25.380 \\
(24.9)\end{array}$ & $\begin{array}{l}16.521-17.320 \\
(16.9)\end{array}$ & Medium & Hexagonal & Prolate & /I & $\begin{array}{l}\text { Zonocolpate } \\
\text { (slit- like) }\end{array}$ & 6 & // & /I & Reticulate & Tectate & $/ /$ & Distinct \\
\hline 7 & $\begin{array}{l}14.620-15.590 \\
(15.105)\end{array}$ & $\begin{array}{l}11.785-12.820 \\
(12.3)\end{array}$ & Small & $\begin{array}{l}\text { Circular- } \\
\text { hexagonal }\end{array}$ & Subprolate & /I & /I & /I & // & /I & // & /I & // & // \\
\hline 8 & $\begin{array}{l}34.897-37.562 \\
(36.229)\end{array}$ & $\begin{array}{l}18.114-21.041 \\
(19.577)\end{array}$ & Large & Semi-circular & Prolate & /1 & // & $22-27$ & // & /l & Psilate & Indistinct & $/ /$ & Indistinct \\
\hline 9 & $\begin{array}{l}16.112-18.928 \\
(17.52)\end{array}$ & $\begin{array}{l}15.125-17.963 \\
(16.544)\end{array}$ & Medium & Triangular & $\begin{array}{l}\text { Prolate- } \\
\text { spheroidal }\end{array}$ & /I & $\begin{array}{l}\text { Zonocolporate } \\
\text { (elliptic) }\end{array}$ & 3 & // & /l & Reticulate & Tectate & // & Distinct \\
\hline 10 & $\begin{array}{l}24.851-25.431 \\
(25.141)\end{array}$ & $\begin{array}{l}23.565-24.162 \\
(23.863)\end{array}$ & /I & Semi-circular & // & /I & // & /I & // & /l & Striate & $\begin{array}{l}\text { Semi- } \\
\text { tectate }\end{array}$ & // & Indistinct \\
\hline 11 & $\begin{array}{l}23.165-24.080 \\
(23.622)\end{array}$ & $\begin{array}{l}22.541-24.323 \\
(23.432)\end{array}$ & /I & Circular & // & Apolar & $\begin{array}{l}\text { Pantoporate } \\
\text { (circular) }\end{array}$ & $25-30$ & // & /I & $\begin{array}{l}\text { Foveate- } \\
\text { micropapilate }\end{array}$ & Tectate & Present & Distinct \\
\hline 12 & $\begin{array}{l}38.564-39.377 \\
(38.970)\end{array}$ & $\begin{array}{l}37.641-38.392 \\
(38.016)\end{array}$ & Large & /I & // & /I & /I & $20-25$ & Present & /I & // & $\begin{array}{l}\text { Semi- } \\
\text { tectate }\end{array}$ & // & Indistinct \\
\hline 13 & $\begin{array}{l}43.652-45.375 \\
(44.513)\end{array}$ & $\begin{array}{l}44.748-45.338 \\
(45.043)\end{array}$ & /I & /I & // & /I & // & $15-20$ & Absent & /l & // & Indistinct & // & /I \\
\hline
\end{tabular}

$(/ /)=$ as pervious; $\mathrm{E}=$ equatorial diameter or width; $\mathrm{P}=$ polar axis or length, $\mathrm{LM}$ : Light microscope, SEM: Scanning electron microscope.

Table 4: Quantitative and qualitative seed micro-morphological characteristics of the studied taxa (LM \& SEM)

\begin{tabular}{|c|c|c|c|c|c|c|c|c|c|c|c|c|c|}
\hline \multirow{2}{*}{$\begin{array}{r}\text { Attributes } \\
\text { Taxa }\end{array}$} & \multirow{2}{*}{ Color } & \multirow[t]{2}{*}{ Shape } & \multirow{2}{*}{$\begin{array}{l}\text { Dimension } \\
(\mathrm{Lx} \mathrm{W}) \mathrm{mm}\end{array}$} & \multicolumn{2}{|l|}{ Hilum } & \multirow{2}{*}{$\begin{array}{l}\text { Surface sculpture } \\
\text { pattern }\end{array}$} & \multirow{2}{*}{$\begin{array}{l}\text { Epidermal cell } \\
\text { shape }\end{array}$} & \multicolumn{4}{|c|}{ Anticlinal wall } & \multicolumn{2}{|c|}{ Periclinal wall } \\
\hline & & & & Position & Level & & & Elevation & Width & Shape & Surface sculpture & Elevation & Surface sculpture \\
\hline 1 & Dark brown & Elliptic & $\begin{array}{l}1.5-1.8 \times 0.8- \\
1\end{array}$ & $\begin{array}{l}\text { Sub- } \\
\text { basal }\end{array}$ & $\begin{array}{l}\text { Semi- } \\
\text { depressed }\end{array}$ & $\begin{array}{l}\text { Tuberculate- } \\
\text { ruminate }\end{array}$ & Polygonal & Raised & Narrow & Straight & Smooth & Depressed & Smooth \\
\hline 2 & // & // & $\begin{array}{l}1.9-2.1 \text { X } 0.8- \\
1\end{array}$ & Basal & Flat & Reticulate & /I & /I & Wide & /I & Tuberculate & // & Obscure \\
\hline 3 & Brown & Oblong ovate & $\begin{array}{l}0.6-0.8 \times 0.3- \\
0.5\end{array}$ & /l & /I & Ruminate & Irregular & Depressed & Narrow & Sinuate & Obscure & Raised & $\begin{array}{l}\text { Striate to } \\
\text { microreticulate }\end{array}$ \\
\hline 4 & Light brown & Orbicular & $\begin{array}{l}0.6-0.8 \times 0.5- \\
0.6\end{array}$ & /I & Depressed & Scalariform & Isodiametric & Raised & Wide & Straight & Smooth & Depressed & Smooth \\
\hline 5 & Black & $\begin{array}{l}\text { Obovate to } \\
\text { orbicular }\end{array}$ & $\begin{array}{cc}1- & 1.5 \mathrm{X} \\
0.9-1.3 & \end{array}$ & II & Flat & Cerebelloid & Polygonal & /I & // & Sinuate & /I & // & Glebulate \\
\hline 6 & Light brown & Oblong ovate & $\begin{array}{l}0.4-0.8 \times 0.1- \\
0.3\end{array}$ & /I & Raised & Rugose & Irregular & /I & // & Straight & Ribbed & // & Smooth \\
\hline 7 & $\begin{array}{l}\text { Yellowish } \\
\text { brown }\end{array}$ & Subglobose & $\begin{array}{l}0.9-1.3 \times 0.8- \\
1.2\end{array}$ & /I & // & Ruminate & /I & /I & /I & Undulate & Smooth & /I & /I \\
\hline 8 & Black & Elliptic & $\begin{array}{l}2.9-3.9 \times 0.5- \\
1.1\end{array}$ & $\begin{array}{l}\text { Sub- } \\
\text { basal }\end{array}$ & // & Reticulate, hairy & Polygonal & Depressed & Narrow & Straight & Obscure & Raised & /I \\
\hline 9 & // & Cuboid & $\begin{array}{l}0.6-0.8 \times 0.4- \\
0.8\end{array}$ & /I & Flat & $\begin{array}{l}\text { Reticulate - } \\
\text { verrucate }\end{array}$ & Polygonal & Raised & // & /I & Smooth & Depressed & Verrucate \\
\hline 10 & Light brown & Elliptic & $\begin{array}{l}1.8-4 \mathrm{X} \\
1.6-1.8\end{array}$ & Basal & Raised & $\begin{array}{l}\text { Reticulate- } \\
\text { scalariform }\end{array}$ & /I & /I & Wide & /I & /I & /I & Smooth \\
\hline 11 & Dark brown & $\begin{array}{l}\text { Reniform to } \\
\text { elliptic }\end{array}$ & $\begin{array}{l}2.9-3.5 \times 1.6- \\
1.9\end{array}$ & /l & /I & Colliculate & $\begin{array}{l}\text { Elongated } \\
\text { polygonal }\end{array}$ & Depressed & Narrow & Sinuate & Smooth & Raised & Granulate \\
\hline 12 & /I & $/ /$ & $\begin{array}{l}1.5-1.8 \times 1.1- \\
1.4\end{array}$ & /I & Depressed & Aculeate- verrucate & Irregular & // & // & Undulate & $1 /$ & $/ /$ & /I \\
\hline 13 & Light brown & // & $\begin{array}{c}1.1- \\
1-1.4\end{array}$ & $1 /$ & /I & Colliculate & $\begin{array}{l}\text { Elongated } \\
\text { polygonal }\end{array}$ & /I & // & Sinuate & /I & // & /I \\
\hline
\end{tabular}

$(/ /)=$ as pervious; $(\mathrm{L})=$ length; $(\mathrm{W})=$ width. $\mathrm{LM}$ : Light microscope, SEM: Scanning electron microscope

obvious seed characters can be used as a confirmatory key that ensure the identification of the studied taxa as follow:

\begin{tabular}{ll}
\hline Distinguishing features & Character/taxa \\
\hline 1a. Black colored seed & 2 \\
1b. Brownish colored & 4 \\
2a. Seed covered with long hairs & Polygala sinaica \\
2b. Seed glabrous & 3 \\
3a. Obovate to orbicular shape, cerebelloid surface sculpture & Hyoscyamus boveanus \\
3b. Cuboid shape, reticulate- verrucate surface sculpture & Primula boveana \\
4a. Reniform shape & 5 \\
4b. Not as above & 7 \\
5a. Aculeate- verrucate surface sculpture & Silene oreosinaica \\
5b. Colliculate surface sculpture & 6 \\
6a. Dark brown seed & Silene leucophylla \\
6b. Light brown & Silene schimperiana \\
7a. Subglobose shape, yellowish brown, ruminate & surface Origanum syriacum \\
sculpture & subsp. sinaicum \\
7b. Ovate, oblong ovate, orbicular or elliptic shape & 8 \\
8a. Orbicularshape, lightbrowncolor,scalariformsurface sculpture & Buffonia multiceps \\
8b. Oblong ovate or elliptic shape & 9
\end{tabular}

\begin{tabular}{ll} 
9a. Oblong ovate shape & 10 \\
9b. Elliptic shape & 11 \\
10a. Rugose surface sculpture & Micromeria serbaliana \\
10b. Ruminate surface sculpture & Ballota kaiseri \\
11a. Tuberculate- ruminate surface sculpture & Anarrhinum pubescens \\
11b. Reticulate surface sculpture & Astragalus fresenii \\
11c. Reticulate- scalariform surface sculpture & Rosa arabica \\
\hline
\end{tabular}

Energy dispersive X-ray (EDX) investigation

The mineral composition of endemic taxa pollen grains and seeds in SKP indicated that 12 elements were represented in both of them (Table 5). Pollen grains and seeds constituted a rich source of mineral elements. Mineral composition and relationship among them for pollen grains indicated that the predominant minerals were carbon $(\mathrm{C})$ and oxygen $(\mathrm{O})$ (in both pollen and seeds of all endemic 


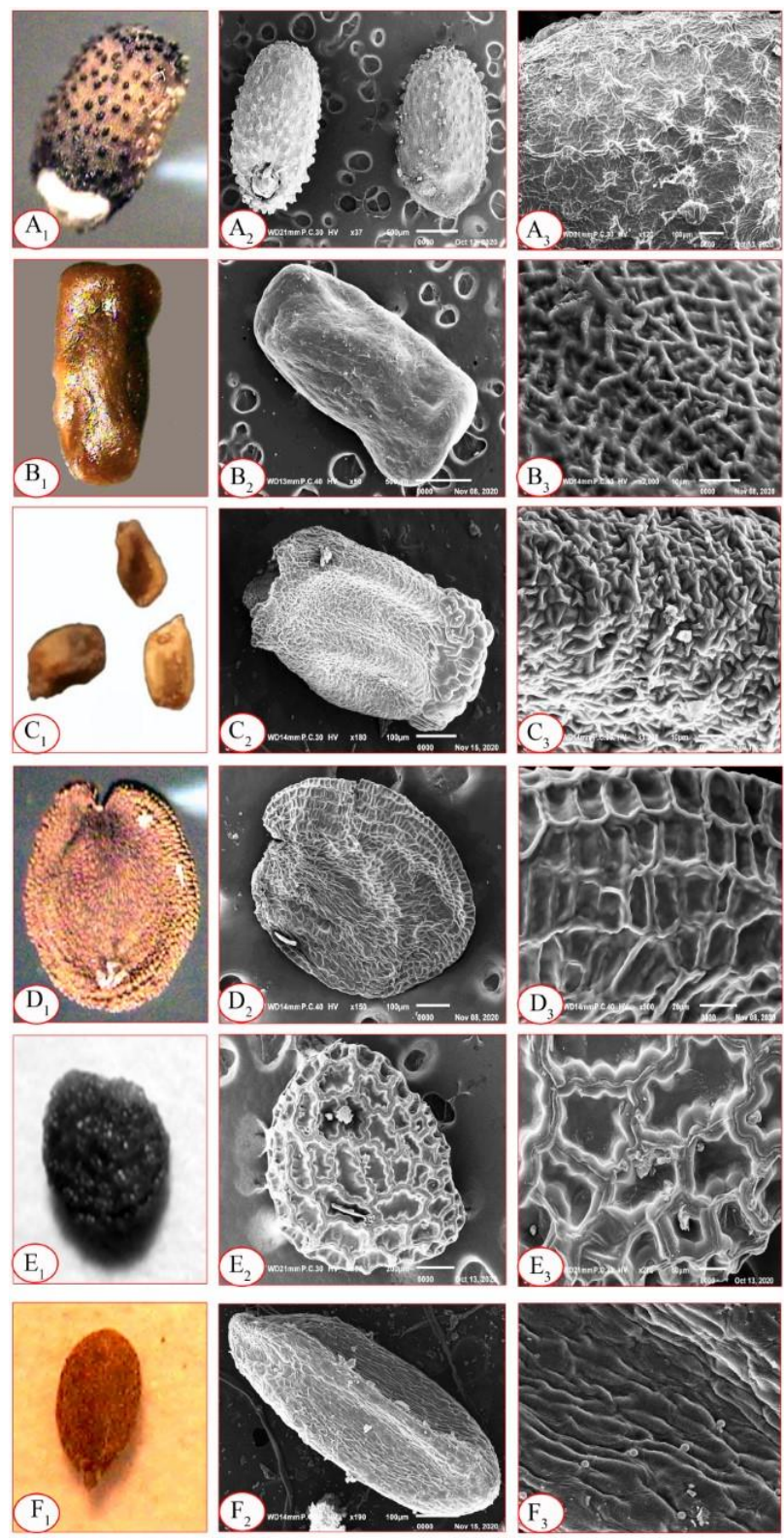

Fig. 3: A-F. Micro-photographs of seeds of the studies taxa (LM \& SEM). (A) Anarrhinum pubescens; dark brown, elliptic, polygonal cell shape, tuberculate- ruminate surface sculpture, (B) Astragalus fresenii; dark brown, elliptic, polygonal cell shape, reticulate surface sculpture, (C) Ballota kaiseri; brown, oblongovate, irregular cell shape, ruminate surface sculpture, (D) Buffonia multiceps; light brown, orbicular, isodiametric cell shape, scalariform surface sculpture, (E) Hyoscyamus boveanus; black, obovate to orbicular, polygonal cell shape, cerebelloid surface sculpture, (F) Micromeria serbaliana; light brown, oblong ovate, irregular cell shape, rugose surface sculpture

taxa in SKP) followed by phosphorous (P), magnesium $(\mathrm{Mg})$ and chloride $(\mathrm{Cl})$, while sodium $(\mathrm{Na})$, silicon $(\mathrm{Si})$ and aluminum $(\mathrm{Al})$ are less represented (they represented only in three studied taxa). C ranged from $54.0 \%$ for pollens of Silene. leucophylla to $73.4 \%$ for pollens of Silene
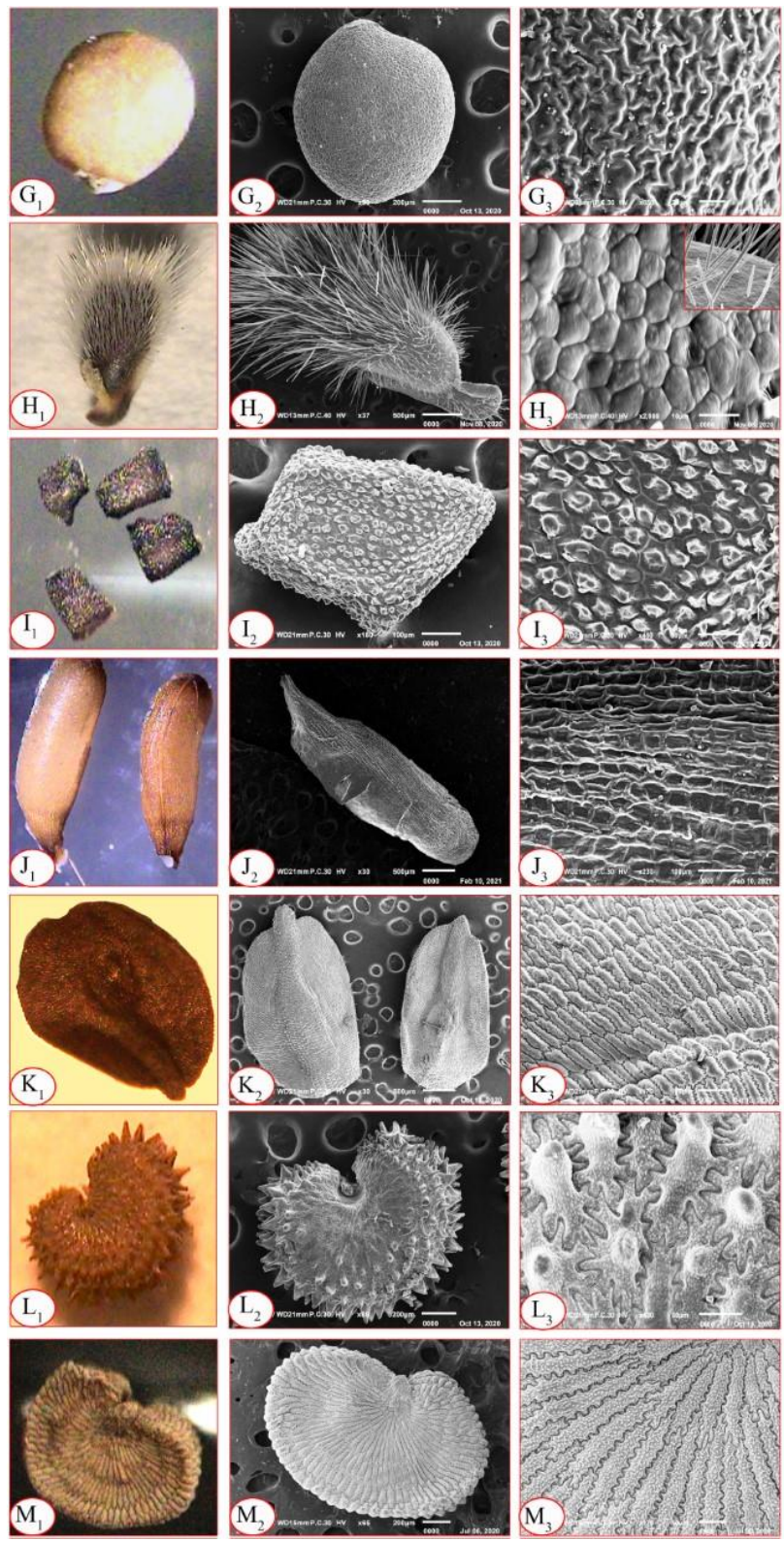

Fig. 3: Cont. G-M. Micro-photographs of seeds of the studies taxa (LM \& SEM). (G) Origanum syriacum subsp. sinaicum; yellowish brown, subglobose, irregular cell shape, ruminate surface sculpture, (H) Polygala sinaica; black, hairy, elliptic, polygonal cell shape, reticulate surface sculpture, (I) Primula boveana; black, cuboid, polygonal cell shape, reticulate- verrucate surface sculpture, (J) Rosa arabica; light brown to brown, elliptic, polygonal cell shape, reticulate- scalariform surface sculpture, (K) Silene leucophylla; dark brown , reniform to elliptic, elongated polygonal cell shape, colliculate surface sculpture, (L) S. oreosinaica; dark brown , reniform, irregular cell shape, aculeate- verrucate surface sculpture, (M) S. schimperiana; light brown, reniform, elongated polygonal cell shape, colliculate surface sculpture

schimperiana, while O ranged from $17.9 \%$ for pollens of Silene oreosinaica to $31.3 \%$ for pollens of Rosa arabica. The percentage for nitrogen $(\mathrm{N})$ varied between 18.4 to 
El-Khalafy et al. / Intl J Agric Biol, Vol 26, No 2, 2021

Table 5: Element presence and their percentage in pollen grains and seeds of the studied taxa by EDX

\begin{tabular}{|c|c|c|c|c|c|c|c|c|c|c|c|c|}
\hline Element Taxa & $\mathrm{C}$ & $\mathrm{N}$ & $\mathrm{O}$ & $\mathrm{Mg}$ & $\mathrm{Al}$ & $\mathrm{P}$ & $\mathrm{S}$ & $\mathrm{Cl}$ & $\mathrm{K}$ & $\mathrm{Ca}$ & $\mathrm{Si}$ & $\mathrm{Na}$ \\
\hline \multicolumn{13}{|l|}{ Pollen } \\
\hline 1 & 55.3 & 20.4 & 23.3 & 0.12 & 0.12 & 0.32 & 0.15 & 0.08 & 0.19 & 0.07 & - & - \\
\hline 2 & 68.1 & - & 30.2 & 0.19 & - & 0.23 & 0.12 & 0.60 & 0.10 & 0.47 & - & - \\
\hline 4 & 72.4 & - & 26.5 & 0.10 & 0.17 & 0.09 & - & 0.13 & 0.24 & 0.14 & 0.24 & - \\
\hline 5 & 67.8 & - & 29.7 & 0.30 & 0.49 & 0.20 & 0.12 & 0.52 & 0.15 & 0.13 & 0.27 & 0.34 \\
\hline 6 & 68.8 & - & 29.9 & 0.21 & - & - & - & 0.49 & 0.04 & 0.36 & - & 0.16 \\
\hline 7 & 72.9 & - & 25.3 & 0.17 & - & 0.26 & 0.29 & 0.15 & 0.37 & 0.35 & - & 0.16 \\
\hline 8 & 56.2 & 21.1 & 22.0 & - & - & 0.24 & 0.11 & - & 0.38 & - & - & - \\
\hline 9 & 55.9 & 18.4 & 24.8 & 0.13 & - & 0.13 & 0.14 & 0.30 & 0.07 & 0.13 & 0.04 & - \\
\hline 10 & 68.0 & - & 31.3 & 0.13 & - & 0.16 & 0.12 & - & 0.26 & 0.08 & - & - \\
\hline 11 & 54.0 & 20.3 & 24.2 & 0.17 & - & 0.37 & 0.15 & 0.17 & 0.63 & - & - & - \\
\hline 12 & 80.6 & - & 17.9 & 0.20 & - & 0.39 & 0.19 & 0.21 & 0.44 & - & - & - \\
\hline 13 & 73.4 & - & 26.1 & - & - & 0.10 & - & 0.13 & 0.30 & 0.07 & & \\
\hline \multicolumn{13}{|l|}{ Seed } \\
\hline 1 & 61.8 & - & 35.5 & 0.32 & 0.69 & 0.18 & 0.12 & - & - & 1.40 & - & - \\
\hline 3 & 41.5 & 13.4 & 43.2 & 0.48 & - & 0.24 & 0.11 & 0.15 & 0.90 & - & - & - \\
\hline 4 & 56.6 & - & 42.5 & - & - & - & - & 0.07 & 0.63 & 0.15 & - & - \\
\hline 5 & 54.3 & - & 44.3 & 0.18 & 0.18 & - & - & 0.17 & 0.13 & 0.38 & 0.14 & - \\
\hline 6 & 55.0 & - & 43.3 & 0.28 & 0.19 & - & - & 0.08 & 0.11 & 0.58 & 0.32 & 0.15 \\
\hline 7 & 54.8 & - & 44.2 & - & 0.39 & - & - & 0.03 & - & 0.57 & - & - \\
\hline 8 & 55.4 & - & 43.4 & 0.13 & - & - & - & - & 0.31 & 0.74 & - & - \\
\hline 9 & 58.2 & - & 40.2 & - & 0.47 & - & - & 0.13 & 0.41 & 0.27 & - & 0.26 \\
\hline 10 & 68.2 & - & 31.7 & - & - & - & - & - & - & 0.14 & - & - \\
\hline 11 & 58.3 & - & 40.6 & 0.17 & - & - & - & - & 0.23 & 0.74 & - & - \\
\hline 12 & 45.5 & 18.2 & 35.0 & - & 0.15 & - & 0.05 & 0.13 & 0.28 & 0.66 & - & - \\
\hline 13 & 42.1 & 23.9 & 33.0 & 0.31 & - & - & - & - & 0.34 & 0.32 & - & - \\
\hline
\end{tabular}

(C) carbon, (N) nitrogen, $(\mathrm{O})$ oxygem, $(\mathrm{Mg})$ magnesium, $(\mathrm{Al})$ aluminum, $(\mathrm{P})$ phosphorus, $(\mathrm{S})$ Sulphur, $(\mathrm{Cl})$ chloride, $(\mathrm{K})$ potassium, $(\mathrm{Ca})$ calcium, $(\mathrm{Si})$ silicon, $(\mathrm{Na})$ sodium

Table 6: The updated list of endemic taxa in SKP in the present study compared to the four previous related studies; 1- Boulous, 2009; 2- Hosni et al. (2013); 3- Zahran et al. (2015); 4- Abdelaal et al. 2018 and 5- present study. ( $\times$ ) refers to exclusion from the endemics while $(\sqrt{ })$ refers to being in the endemics

\begin{tabular}{|c|c|}
\hline \multicolumn{2}{|r|}{12345} \\
\hline \multicolumn{2}{|l|}{$\begin{array}{l}\text { Taxa } \\
\text { Anarrhinum pubescens, Astraglus fresenii, Ballota kaiseri, Buffonia } \\
\text { syriacum L }\end{array}$} \\
\hline Silene shimperiana & $\sqrt{ } \times \sqrt{ } \times \sqrt{ }$ \\
\hline Polygala sinaica var. sinaica & $\sqrt{ } \sqrt{ } \times \sqrt{ }$ \\
\hline Euphorbia obovata Decne. & $\sqrt{ } \sqrt{ } \sqrt{ } x$ \\
\hline Phlomis aurea Decne. & $\sqrt{ } \sqrt{ } \sqrt{ } x$ \\
\hline Pterocephalus arabicus Boiss. & $\sqrt{ } \sqrt{ } \times x$ \\
\hline Veronica kaiseri Täckh. & $\sqrt{ } \sqrt{ } \times x$ \\
\hline Silene odontopetala Fenzl var. congesta (Boiss.) Melzh. & $\sqrt{ } \sqrt{ } \times x \times$ \\
\hline Teucrium leucocladum Boiss. subsp. sinaicum Danin & $x \sqrt{ } \times \sqrt{ } \times$ \\
\hline Teucrium leucocladum Boiss. subsp. leucocladum var. glandulosum Danin & $\times \sqrt{ } \times \times \times$ \\
\hline Phagnalon nitidum Fresen. & $\sqrt{ } \times \times \times \times$ \\
\hline Plantago sinaica (Barn.) Decne. & $\sqrt{ } \times x \times x$ \\
\hline Euphorbia sanctae-catharinae Fayed & $x \sqrt{ } \times x \times$ \\
\hline Kickxia macilenta & $\times \sqrt{ } \times x \times$ \\
\hline Nepeta septemcrenata Benth. & $\times \sqrt{ } \times \times \times$ \\
\hline Veronica musa Täckh. \& Hadidi & $\times \sqrt{ } \times x$ \\
\hline
\end{tabular}

$21.2 \%$ and calcium $(\mathrm{Ca})$ from 0.06 to $.47 \%$. On the other hand, mineral composition and relationship among them for seeds indicated the predominant minerals were $\mathrm{C}$ and $\mathrm{O}$ (represented in pollen and seeds of all endemic taxa in SKP) followed by potassium $(\mathrm{K})$ and $\mathrm{Ca}$, while $\mathrm{P}, \mathrm{Na}$ and $\mathrm{Si}$ are less represented (they represented by only two taxa). The carbon ranged from $42.1 \%$ for $S$. schimperiana to $68.2 \%$ for $R$. arabica, while $\mathrm{O}$ ranged from $31.7 \%$ for seeds of $R$. arabica to $44.3 \%$ for seeds of Hyoscyamus boveanus. $\mathrm{N}$ percentage varied between 13.4 and $23.9 \%$ and $\mathrm{Ca}$ from 0.14 to $1.4 \%$.

\section{Discussion}

Endemism is the occurrence of a taxon to a restricted defined country, while the narrow-distributed taxa restricted to a limited area in the country are steno-endemics (Gaston 1994). Thirteen taxa of vascular plants, belonged to 11 genera and eight families, were recorded in SKP in the present study comparing with the previous survey of Boulos (2009) who reported 20 endemic taxa, Hosni et al. (2013) and Zahran et al. (2015) recorded 17 taxa, while Abdelaal et al. (2018) recorded 14 taxa. In the present study, eleven taxa 
were common between the four previously mentioned studies. In addition, Polygala sinaica var. sinaica and $S$. schimperiana were only recorded by Boulos (2009) and Zahran et al. (2015). As shown in Table 6, the variation in the number of endemic taxa from those of the previous studies was based on the presence of endemic taxa in a country other than Egypt. Additional records were collected from literature, scientific websites and global databases with specific localities, coordinates, photos or herbarium specimens. Two taxa were excluded from the endemism of SKP for the first time; Euphorbia obovata was recorded in Iran, Palestine, Syria and India (Websites 6 and 13; Barnet 2002) and Phlomis aurae in Jordan and Saudi Arabia (Websites 7 and 6; Al-Eisawi and Al-Khader 2007).

In angiosperms, sex is a quantitative phenomenon that can be measured on a continuous scale between strictly male and female extremes (Lloyd 1980). All endemic taxa in SKP were hermaphrodite. The preponderance of the hermaphroditic species (bisexual) is a common feature in most world floras. The ecological and evolutionary significance of bisexuality was emphasized by Baker and Hurd (1968). They suggested that the co-evolution of hermaphroditic flowers with animal pollination might be an important advancement by early angiosperms since pollenproducing and pollen-receiving organs in the same flower allowed for efficient simultaneous deposition and removal of pollen (Baker and Hurd 1968). Moreover, most dioecious species are animal pollinated (Bawa 1980); though in temperate and arid zones many dioecious species are windpollinated (Freeman et al. 1980; Hultine et al. 2007).

In general, the period from March to May (spring season) was characterized by the highest number of flowering endemic taxa in SKP. In Egypt, the highest humid period of the year extends from November to April, associated with low temperature and evaporation; therefore, much favorable soil moisture. During this period, the plants start their growth, reaching the flowering and fruiting stages in March, April and May, respectively (Burnie et al. 2004; Heneidy 2010). In the present study, the dispersal types of the endemic were assessed using the system of Dansereau and Lems (1957), which distinguished dispersal types primarily by the morphology of the diaspora. Wide distribution of ballochoric plants may be due to their explosive nature, which is often related to rapid desiccation, and hence efficient local seed dispersal.

Pollen morphological characters in some members of endemic and near-endemic taxa in Sinai have been recorded by Shehata and Kamel (2007), Ibrahim (2015) and ElGhamery et al. (2018). From the foregoing data, the pollen morphological characters are considered diagnostic at the specific level among the studied taxa.

All the examined pollen grains are radially symmetric. All pollen grains are isopolar, except Buffonia multiceps, S. leucophylla, S. oreosinaica, S. schimperiana (belonging to Caryophyllaceae) which are a polar. Such result is in accordance with Rabei et al. (2016) and other previous studies on this family (Yildiz 2005), but disagrees with ElGhamery et al. (2018) since they reported that all endemic taxa in their study are isopolar. The shape class of the examined pollen grains is subprolate in $S$. oreosinaica and Origanum syriacum subsp. sinaicum; prolate in Anarrhinum pubescens, Ballota kaiseri, Micromeria serbaliana and $P$. sinaica; while prolate-spheroidal in the rest of the studied taxa. They took generally triangular, hexagonal, circularhexagonal, semi- circular or circular in polar view.

The present pollen data showed considerable variations in their measurable characters viz. polar axis (14.717 $\mu \mathrm{m}$ to $44.513 \mu \mathrm{m})$ and equatorial axis $(9.3115 \mu \mathrm{m}$ to $45.043 \mu \mathrm{m})$ in $A$. pubescens and $S$. schimperiana, respectively. Pollen grains apertures showed variations among the studied taxa. The pantoporate grain with 10- 30 circular pores were observed in B. multiceps, S. leucophylla, $S$. oreosinaica and $S$. schimperiana (operculum detected in B. multiceps and $S$. oreosinaica only and considered a diagnostic character) and the trizonocolporate grain in $A$. pubescens, $A$. fresenii, $H$. boveanus and $R$. arabica (margo detected in $A$. pubescens and $A$. fresenii only and considered a diagnostic character). However, $M$. serbaliana and $O$. syriacum subsp. sinaicum can clearly be delimited from the other studied taxa by having hexacolpate grain and $B$. kaiseri by trizonocolpate grain. Moreover, stephanocolpate pollen grain with 22-27 colpi recorded only in $P$. sinaica which is considered a diagnostic character in contrast with El-Ghamery et al. (2018) who described it as polycolporate pollen. The present finding has been previously reported in some endemic taxa or generally of their families in some studies such as Yildiz (2005); Pinar et al. (2009); Banks et al. (2008); Bazarragchaa et al. (2012); Osman (2012); ElGhamery et al. (2018) and Shiha (2020).

Out of the present pollen morphological characters, seven types of exine sculpture have been observed and considered diagnostic at specific level viz, foveatemicropapillate in three studied Silene species that distinguished them from other studied taxa, reticulate exine in A. fresenii, $M$. serbaliana, $O$. syriacum subsp. sinaicum and $P$. boveana, reticulat foveolate in $A$. pubescens, reticulate-perforate in $B$. kaiseri, striate in $R$. arabica, striateperforate in $H$. boveanus. However, B. multiceps is delimited from other taxa by aculeate exine sculpture. Moreover, $P$. sinaica is distinguished by psilate exine sculpture. Four taxa belonging to Caryophyllaceae are characterized by the presence of annulus which is absent in the rest of the studied taxa. These findings corroborate with Yildiz (2005); Krachai et al. (2009); Pinar et al. (2009); Osman (2012); Mostafavi and Mehregan (2014); El-Ghamery et al. (2018) and Shiha (2020).

The examined seeds of the studied taxa showed significant variation in color, shape, epidermal cell shape, external surface sculpture pattern and periclinal wall sculpture. The seed color was brown (light, yellowish or dark) in most studied taxa except in $H$. boveanus and $P$. boveana where the seed color was black. Moreover, the 
seed was black and covered with long, unicellular, shiny hairs in $P$. sinaica which distinguished it from other taxa. Weitz et al. (1993) and Aydin (2019) reported the presence of such trichomes in their studies. The smallest seeds were measured in $M$. serbaliana $(0.4-0.8 \times 0.1-0.3 \mathrm{~mm})$, while the largest in $S$. leucophylla $(2.9-3.5 \times 1.6-1.9 \mathrm{~mm})$. The seeds exhibited various shapes: the most obvious was the cuboid shape in $P$. boveana, reniform in the three studied Silene species. Elliptic shape, oblong ovate, obovate and subglobose also were recorded. According to Hosny and Zareh (1992/1993) and Ibrahim (2015), the forgoing data reported the reniform seed shape in Silene species. Rabei et al. (2016) studied the seed of S. oreosinaica and also described it as a reniform shape. B. multiceps a member of Caryophyllaceae, have been studied as an orbicular shape in agreement with El-Ghamery et al. (2018).

The fascinating character is the surface sculpture pattern where numerous types were recorded. These were cerebelloid in $H$. boveanus, scalariforn in B. multiceps, tuberculate- ruminate in $A$. pubescens, rugose in $M$. serbaliana, ruminate in $B$. kaiseri and $O$. syriacum subsp. sinaicum, reticulate in $A$. fresenii and $P$. sinaica, reticulateverrucate in $P$. boveana, reticulate- scalariform in $R$. arabica, acileate- verrucate in $S$. oreosinaica and colliculate in S. leucophylla and S. schimperiana. The epidermal cell is arranged in three shape viz, isodiametric, polygonal and irregular. The anticlinal walls were undulate in $O$. syriacum subsp. sinaicum and $S$. oreosinaica, sinuate in B. kaiseri, $H$. boveanus, $S$. leucophylla and $S$. schimperiana or straight in the rest of the studied taxa. Another diagnostic character in addition to the sculpture pattern is the periclinal wall surface sculpture as it was glebulate in $H$. boveanus, striatemicroreticulate in $B$. kaiseri, verrucate in $P$. boveana, granulate in the three studied Silene species, obscure in $A$. fresenii or smooth in the rest of the studied taxa. The present study considered the first description of seeds in A. fresenii, B. kaiseri and M. serbaliana. However, their characteristics followed the general characters of their genera. Similar types of seed surface sculpture patterns in other species of Astragalus were reported by Ekici et al. (2005); Vural et al. (2008) and Shemetova et al. (2018). The reticulate, rugose, ruminate and smooth slightly tuberculate nutlet surface ornamentation were recorded by Kamel (2014) within the studied taxa of Lamiaceae.

The present study indicated that pollen grains and seeds of endemic taxa in SKP had high percentages of C, O, $\mathrm{P}, \mathrm{Mg}, \mathrm{N}$ and $\mathrm{Ca}$. Boughediri (1991) found that phosphorus and potassium had the highest values for most date palm cultivars. $\mathrm{K}$ also appears to dominate in the studied taxa belonging to Plumerioideae and Cerberoideae, while $\mathrm{Ca}$ was equal or dominant in the studied taxa of Apocynoideae (Wolter and Nilsson 1990). The consistent appearance of $\mathrm{P}$ at the aperture area of pollen of Hordeum vulgare and Sesamus indicum was attributed as one factor regulating the regulate the uptake of water and rapid growth of pollen (Rehman et al. 2002, 2004). Bacha et al. (1997) found that pollen grains of date palm contained the highest concentrations of N. Stanley and Linskens (1974) reported that high mineral contents of pollen might be due to the high mineral content in the soil horizons in dry areas where this species grows. The highest percentages of $\mathrm{C}$ and $\mathrm{O}$ may due to they represented as organic matter in these taxa, since they have been classified as essential elements for the plants (Mengel and Kirkby 1982). Variations in the composition of taxa pollens and seeds reflect the differences in the floral origin of pollen and the plant growth conditions such as soil and geographic origin (Stanley 1971; Hassan 2011). The capacity of the parent plant to accumulate salts in the pollen and seeds is also related to the species. Moreover, Ca plays a vital role in plant growth and development cycles such as pollen tube growth and fertilization (Aly 2018). Ca also plays a role in determining the direction of pollen tube growth and has an essential signaling, physiological, and regulatory role during sexual reproduction in flowering plants (Ge et al. 2007; Prajapati and Jain 2010).

\section{Conclusion}

Results of this study were helpful in the authentication and identification of the endemic taxa in SKP. Also, the examined characteristics were useful in the identification and discrimination between the studied taxa. However, some of these taxa need further investigation to verify their exclusion from endemism (B. multiceps, $H$. boveanus, $S$. shimperiana and $P$. sinaica var. sinaica). These taxa are recorded in the countries other than Egypt but without coordinates, photos or herbarium sheets. Therefore, these taxa are not excluded from the endemism in the present study.

\section{Acknowledgements}

This research did not receive any specific grant from funding agencies in the public, commercial, or not-for-profit sectors.

\section{Author Contributions}

Mohamed M. El-Khalafy designed the colored plates, acquisition, analysis and interpretation of results; drafted, revised and approved the final version of the manuscript for submission; Dalia A. Ahmed, Kamal H. Shaltout and Yassin M. Al-Sodany conceived and interpreted the results; revised the article and approved the final version for submission; Soliman A. Haroun planned the study revised the article and approved the final version to be submitted; Mohamed A. Salem prepared initial draft, revised the script, designed the colored plates, analysed and interpreted results and approved the final version to be submitted.

\section{Conflict of Interests}

All of the authors confirm that there are no conflicts of interest. 


\section{Data Availability}

Please contact the authors for data requests.

\section{Ethics Approval}

Not applicable in this paper

\section{References}

Abdelaal M, M Fois, G Fenu, G Bacchetta (2018). Critical checklist of the endemic vascular Flora of Egypt. Phytotaxa 360:019-034

Al-Eisawi DM, IA Al-Khader (2007). Checklist of Plants of the Hashemite Kingdom of Jordan. Available at: http://ww2.odu. edu/ lmusselm/plant/jordan/index.php (Accessed: May 2017)

Aly HS (2018). Evaluation of pollen grains germination, viability and chemical composition of some date palm males. Middle East J Agric Res 7:235-247

Angiosperm Phylogeny Group (2016). An update of the Angiosperm Phylogeny Group classification for the orders and families of flowering plants: APG IV. Bot J Linn Soc 181:1-20

Aydın ZU (2019). Comparative capsule and seed morphology of Polygala L. (Polygalaceae) species in Turkey with implications for taxonomy, Plant Biosyst 154:189-196

Bacha MA, MA Ali, FA Farahat (1997). Chemical composition of pollen grains of some date palm males grown in Riyadh, Saudi Arabia. Arab Gulf J Scientific Res 15:783-803

Baker H, P Hurd Jr (1968). Intrafloral ecology. Ann Rev Entmol 13:385-414

Banks H, B Klitgaard, F Klaxton, C Felix, F Crane (2008). Pollen morphology of family Polygonaceae (Fabales). Bot J Linn Soc $156: 253-289$

Barnet R (2002). All Kinds of Scented Wood: Wood and Woodworking in the Bible. Xulon Press, Maitland, Florida, USA

Barthlott W (1981). Epidermal and seed surface characters of plants: systematic applicability and some evolutionary aspects. Nord J Bot $1: 345-355$

Barthlott W (1984). Microstructural features of seed surface. In: Current Concepts in Plant Taxonomy. Heywood VH, DM Moore (Eds.) Academic Press, London, UK

Bawa K (1980). Evolution of dioecy in flowering plants. Anпи Rev Ecol Syst 11:15-39

Bazarragchaa B, LS Myoung, LH Yuon (2012). Pollen morphology of the family Lamiaceae in Mongolia. $J$ Kor Nat 5:169-179

Boughediri L (1991). Mineral composition of the exine of two male date palms (Phoenix dactylifera L.). Grana 30:525-527

Boulos L (1999-2005). Flora of Egypt (four volumes). Al-Hadara Publishing, Cairo, Egypt

Boulos L (2009). Flora of Egypt Checklist. Revised Annotated Edition. AlHadara Publishing, Cairo, Egypt

Brooks T, R Mittermeier, GDA Fonseca, J Gerlach, M Hoffmann, J Lamoreux, C Mittermeier, J Pilgrim, A Rodrigues (2006). Global biodiversity conservation priorities. Science 313:58-61

Burnie G, S Forrester, S Guest, M Harmony, P Lavarack, M Letgett, R McDonald, S Macoboy, B Molyneux, D Moodie, J Moore, D Newman, T North, K Piennaar, G Purdy, J Silk S Ryan, G Schien (2004). Botany. Ullmann Press, China

Danin A (1986). Flora and vegetation of Sinai. Proc $R$ Soc Edinb 89B:159-168

Dansereau P, K Lems (1957). The grading of dispersal types in plant communities and their ecological significance. Inst Bot L'Univ Montrèal 71:1-52

Ekici M, D Yuzbaşıŏlu, Z Aytac (2005). Morphology, pollen, seed structure and karyological study on Astragalus ovalis Boiss. and Balansa (sect. Ammodendron) in Turkey. Intl J Bot 1:74-78

El-Ghamery AA, HA Hosni, AM Sadek (2018). Pollen and seed morphology of some endemic taxa in Saint Catherine, Sinai, Egypt. Taeckholmia 38:40-60
El-Hadidi MN, A Fayed (1994/1995). Materials for excursion flora of Egypt (EFE). Taeckholmia 15:1-233

El-Hadidi M, H Hosni (2000). Conservation and Threats. In: Flora Aegyptiaca 105-150, El-Hadidi MN (Ed.). Cairo University Herbarium, the Palm Press, Cairo, Egypt

El-khalafy M (2018). Red list of the endemic and near endemic plant species in Egypt. M.Sc Thesis, Tanta University, Tanta, Egypt

Embabi N (2018). Geographic regions of Egypt. In: Landscapes and Landforms of Egypt. World Geomorphological Landscapes, Springer, Cham, Switzerland

Erdtman G (1952). Pollen Morphology and Plant Taxonomy Angiosperms. Almqvist and Wiksell, Stockholm, Sweden

Freeman D, K Harper, W Ostler (1980). Ecology of plant dioecy in the intermountain region of Western North America and California. Oecologia 44:410-417

Gaston K (1994). Rarity. Chapman \& Hall, London, UK

Ge L, H Tian, S Russell (2007). Calcium function and distribution during fertilization in angiosperms. Amer J Bot 94:1046-1060

Hassan HMM (2011). Chemical composition and nutritional value of palm pollen grains. Glob J Biotechnol Biochem 6:1-7

Heneidy S (2010). Plant Altas "The Botanic Gaden (Alex)". Faculty of Science, Alexandria University, Monsha'a Al Maaref, Alexandria, Egypt

Hesse M, H Halbritter, R Zetter, M Weber, R Buchner, A Frosch-Radivo, S Ulrich (2009). Pollen Terminology. An illustrated Handbook. Springer, Wien-New York, USA

Hosni H, A Hosny, E Shamso, R Hamdy (2013). Endemic and nearendemic taxa in the flora of Egypt. Egypt J Bot 53:357-383

Hosny AI, MM Zareh (1992/1993). Taxonomic studies of Silenoideae (Caryphyllaceae) in Egypt. 2. Taxonomic significance of seed characters in genus Silene $\mathrm{L}$. with special reference to seed sculpture. Taeckholmia 14:37-44

Hultine K, S Bush, A West, J Ehleringer (2007). Population structure, physiology and ecohydrological impacts of dioecious riparian tree species of Western North America. Oecologia 154:85-93

Ibraheem M (2012). Natural Protectorates in Egypt: Related Laws, Legislations and Conventions. Printshop of Akhbar Alyoum: Cairo, Egypt

Ibrahim NO (2015). Taxonomical and molecular characterization of endemic species at Saint Catherine protectorate, Sinai- Egypt. PhD Thesis. Mansoura University, Egypt

Ibrahim K, H Hosni, P Peterson (2016). Grasses of Egypt. Simthsonian Institution. Scholarly Press, Washington DC, USA

Kamel WM (2014). Nutlet morphology and its taxonomic implication in some taxa of Lamiaceae in Egypt, Taeckholmia 34:101-127

Kaya A, F Satıl, M Aslan (2016). Seed morphology of the genus Hyoscyamus L. in Turkey and its systematic significance. Acta Microsc 25:48-55

Krachai P, P Chantaranothai, N piwpuan (2009). Pollen characteristics of Polygala, Salomonia and Xanthophyllum (Polygalaceae) in Thailand. Nat Hist J Chulalongkron Univ 9:27-34

Krzywinski K, K Faegri, J Iversen, P Kaland (1989). Textbook of Pollen Analysis, $4^{\text {th }}$ edn. John Wiley \& Sons, New York, USA

Lima R, V Souza, M De Siqueira, H Steega (2020). Defining endemism levels for biodiversity conservation: Tree species in the Atlantic Forest hotspot. Biol Conserv 252:108825

Lloyd D (1980). Sexual Strategies in Plants. I: An hypothesis of serial adjustment of maternal investment during one reproductive session. New Phytol 86:69-79

Lomonosova MN (2009). Morphology and ultrasculpture of the seed surface in the subfamily Suaedoideae (Chenopodiaceae). Bot J 5:736-744

Lott JN, JS Greenwood, CM Vollmer (1982). Mineral reserves in castor beans: the dry seed. Plant Physiol 69:829-833

Lott JN (1984). Accumulation of seed reserves of phosphorus and other minerals. In: Seed Physiology, Vol 1, pp:139-166. Murray DR (Ed.). Academic Press, Sydney, Australia

Mengel K, EA Kirkby (1982). Principles of Plant Nutrition, $3^{\text {rd }}$ edn, International Potash Institute, Worbalaufen- Bern, Switzerland

Moore PD, JA Webb, ME Collinson (1991). Pollen Analysis, $2^{\text {nd }}$ edn Blackwell Scientific Publications, Oxford, UK 
Mostafavi G, I Mehregan (2014). Pollen Micro-morphology of the Minuartia species (Caryophyllaceae) in Iran. Intl J Modern Bot 4:8-21

Ockenden IE, JN Lott (1988). Mineral storage in Cucurbita embryos. I. Calcium storage in relation to embryo size. Can J Bot 66:1477-1481

Osman AK (2012). Comparative anatomical and palynological studies on genus Ballota (Lamiaceae) from Egypt. $J$ Med Plants Res 6:5797-5812

Pinar NM, M Ekici, Z Ayatc, H Akan, T Ceter, S Alan (2009). Pollen morphology of Astragalus L. sect. Onobrychoidei DC. (Fabaceae) in Turkey. Turk J Bot 291-303

Pocock SA, G Vasanthy (1986). EDS analysis of pollen wall surfaces of Vernonia monosis (Asteraceae) and pollen-soil concentration of elements, Geophytology 16:37-53

Prajapati PP, BK Jain (2010). Effect of sucrose, boron, calcium, magnesium and nitrate during in vitro pollen germination in Luffa aegyptica Mill., Prajna. J Pure Appl Sci 18:5-8

Punt W, PP Hoen, S Blackmore, S Nilsson, A Le Thomas (2007). Glossary of pollen and spore terminology. Rev Palaeobot Palyn 143:1-81

Rabei S, M Nada, I El Gamal (2016). Bio-systematic study on the endemic Silene oerosinaica from Sinai, Egypt. Sci J Damietta, Fac Sci 6:183-188

Rehman S, KJ Lee, ES Rha, SJ Yun, JK Kim (2002). Mechanisms involved in rapid swelling of sesame (Sesamun indicum L.) pollen. New Zeal J Crop Hortic Sci 30:203-213

Rehman S, ES Rha, M Ashraf, KJ Lee, SJ Yun, YG Kwak NM Yoo, JK Kim (2004). Does barley (Hordeum vulgare L.) pollen swell in fractions of a second? Plant Sci 167:137-142

Rehman S, M Jamil, ES Rha, M Ashraf, SJ Yun (2008). Is pine (Pinus densiflora) pollen another example of potassium accumulation at sulcus area? Pak J Bot 40:495-500

Shaltout K, D Ahmed, M Diab, M El-Khalafy (2018). Re-assessment of the near-endemic taxa in the Egyptian flora. Taeckholmia 38:61-83

Shehata AA, WM Kamel (2007). A contribution to the palynological studies of the endemic flora of Sinai, Egypt. Bot Stec 11:9-16

Shemetova T, A Erst, W Wang, K Xiang, C Vural, Z Aytaç (2018). Seed morphology of the genus Astragalus L. from North Asia, Turk J Bot 42:710-721

Shiha MA (2020). Taxonomic significance of stamens and pollen morphology of some selected taxa of Primulaceae in Egypt, Taeckholmia 40:1-11
Stanley RG, HF Linskens (1974). Pollen: Biology, Chemistry and Management. Springer-Verlag, New York, USA

Stanley RG (1971). Pollen chemistry and tube growth in pollen. In: Development and Physiology, pp:131-155. Heslop-Harrison J (Ed.). Butterworths, London, UK

Stearn WT (1992). Botanical Latin, $4^{\text {th }}$ edn. David and Charles Publication, London, UK

Svetlova AA (2008). Ultrasculpture of the seeds and anatomical structure of seed coat in species of different sections of the genus Linum (Linaceae). Bot $J$ 11:1760-1771

Täckholm V, G Täckholm (1941). Flora of Egypt: Volume I, Bull Fac Sci Fuoad I Univ 17:1-574

Täckholm V, M Drar (1950-1969). Flora of Egypt: Volume II- IV, Bull Fac Sci Fuoad I Univ 28:30-36

Täckholm V (1974). Students' Flora of Egypt, $2^{\text {nd }}$ edn. Cairo University, Egypt

Taifour H, A El-Oqlah (2014). Jordan Plant Red List. Royal Botanic Garden, Jordan

Takhtajan AL (1980). Outline of the classification of flowering plants (Magnoliophyta). Bot Rev 46:226-339

Vural C, M Ekici, H Akan (2008). Seed morphology and its systematic implication for genus Astragalus L. sections Onobrychoidei DC, Uliginosi Gray and Ornithopodium Bunge (Fabaceae). Plant Syst Evol 274:255-263

Weitz FM, MJ Isaacs, CT Johnson (1993). Seed-coat characteristics of selected southern African species of Polygala L. (Polygalaceae). S Afr J Bot 59:592-596

Wolter M, S Nilsson (1990). Energy dispersive X-ray (EDX) investigations on some Apocynaceae pollen. Grana 29:213-217

Yao YF, XY Song, AH Wortley, YF Wang, S Blackmore, CS Li (2017). Pollen-based reconstruction of vegetational and climatic change over the past $\sim 30 \mathrm{ka}$ at Shudu Lake in the Hengduan Mountains of Yunnan, southwestern China. PLoS One 12; Article e0171967

Yildiz K (2005). A palynological investigation on Silene L (Caryophyllaceae) species distributed in North Cyprus and West Anatolia. CBÜ Fen Bilimleri Dergisi 1:61-71

Zahran M, M Amer, A Aflah, O. Ghaly (2015). Endemic species in Sinai Peninsula, Egypt, with particular reference to Saint Katherine Protectorate: I- Ecological features. J Environ Sci 44:589-609

Zoric L, L Merkulov, J Lukovic, P Boza (2010). Comparative seed morphology of Trifolium L. species (Fabaceae). Period Biol 112:263-272 